\title{
Mixed convection of micropolar fluid on a permeable stretching surface of another quiescent fluid
}

\author{
Nurazleen Abdul Majid ${ }^{a}$, Nurul Farahain Mohammad ${ }^{a,{ }^{*}}$, Abdul Rahman Mohd Kasim ${ }^{\mathrm{b}}$, Sharidan \\ Shafie $^{\mathrm{c}}$ \\ a Department of Computational \& Theoretical Sciences, Kulliyyah of Science, International Islamic University Malaysia, 25200 Kuantan, Pahang, \\ Malaysia \\ ${ }^{b}$ Applied \& Industrial Mathematics Research Group, Faculty of Industrial Sciences \& Technology, University Malaysia Pahang, 26300 Gambang, \\ Pahang \\ c Department of Mathematical Sciences, Faculty of Science, University Teknologi Malaysia, 81310 Skudai, Johor, Malaysia
}

* Corresponding author: farahain@iium.edu.my

Article history

Received 23 August 2019

Revised 28 January 2020

Accepted 1 Mac 2020

Published Online 26 August 2020

\begin{abstract}
In recent decades, micropolar fluid has been one of the major interesting research subjects due to the numerous applications such as blood, paint, body fluid, polymers, colloidal fluid and suspension fluid. However, the behavior of micropolar fluid flow over a permeable stretching surface of another quiescent fluid with a heavier density of micropolar fluid under the condition of mixed convection is still unknown. Thus, the current work aims to investigate numerically the mixed convection of micropolar fluid flow over a permeable stretching surface of another quiescent fluid. In this research, the similarity transformation is implemented to reduce the boundary layer governing equations from partial differential equations to a system of nonlinear ordinary differential equations. Then, this model is solved numerically using shooting technique with Runge-Kutta-Gill method and applied in Jupyter Notebook using Python 3 language. The behavior of micropolar fluid in terms of velocity, skin friction, microrotation and temperature are analyzed.
\end{abstract}

Keywords: Micropolar fluid, quiescent fluids, stretching, mixed convection, permeable

\section{INTRODUCTION}

Micropolar fluid is one of the non-Newtonian fluid that is often being the subject of research due its ability insolving the fluid containing suspended particle such as animal blood, body fluid, colloidal fluid, magnetic fluids, cloud with dust, muddy fluids and polymer that cannot be explained by Navier-Stokes equation. Eringen (1965) was the first researcher who proposed the theory of micropolar fluid. Since then, many researchers have expanded his work to other different geometries such as stretching or shrinking surface and permeable surface. Currently, micropolar fluid is being used to model the blood flow through the tapered artery (Haghighi \& Asl, 2015) and chyme transport in the human intestinal system (Kiran et al. , 2017).

The topic of heat transfer in boundary layer flow is one of the growing research disciplines as it is widely used especially in industry such as transpiration cooling, drag reduction, thermal recovery of oil, the design of thrust bearings and radial diffusers, material drying and laser pulse heating (Attia, 2008). Mixed convection is one of the heat transfer mechanism that happens when the impact of buoyancy force in forced convection or the impact of forced flow in free convection is present. Earlier, Takhar et al. (1998) studied micropolar fluid flow over a stretching sheet under the effect of mixed convection. They found that higher suction slow down the velocity and decrease the boundary layer thickness. Ishak et al. (2007) carried out numerical study of mixed convection of the stagnation point flow towards a stretching vertical permeable sheet. They reported that heat transfer from the surface increased with the presence of suction. Next, Ishak et al. (2008) investigated mixed convection of micropolar fluid towards a stretching sheet on a stagnation point. From this study, the result showed that with the increment of Prandtl number Pr, the thermal boundary layer thickness decreased.

Nevertheless, the system involving two fluids also exists. Wang (1988) proposed the mathematical model of stagnation flow on the surface of a quiescent fluid. Then, Reza \& Gupta (2012) discover the relationship of magnetic effect on the electrically conducting fluid in the surface of another quiescent fluid. Years later, Reza et al. (2017) performed an analysis of stagnation point flow and heat transfer for viscoelastic fluid impinging on a quiescent fluid. The stagnation-point flow of a fluid on a shrinking surface of another quiescent fluid was attempted by Rohni et al. (2015). In other study, Isa \& Mohammad (2017) researched the boundary layer flow on a stretching sheet of another quiescent fluid. They discovered that increasing suction parameter increased the velocity of upper fluid while decreased the velocity of lower fluid. Very recently, Majid et al., (2019) studied the effect of constant heat flux on forced convective micropolar fluid flow over a surface of another quiescent fluid. They mentioned that with the increment of Pr number, the temperatures of both upper and lower fluid decrease. From the previous works mentioned, the mixed convection of micropolar fluid flow on another quiescent fluid is not investigated yet. Thus, this research paper intended to solve this problem. 


\section{MATHEMATICAL MODEL}

Consider an incompressible micropolar fluid of density $\rho_{1}$, dynamic viscosity $\mu_{1}$, vortex viscosity $\kappa_{1}$, spin-gradient viscosity $\gamma_{1}$, acceleration due to gravity $g_{1}$ and microinertia density $j_{1}$ impinging orthogonally on a permeable stretching or shrinking surface of another quiescent, heavier incompressible micropolar fluid of density $\rho_{2}$, dynamic viscosity $\mu_{2}$, vortex viscosity $\kappa_{2}$, spin-gradient viscosity $\gamma_{2}$, acceleration due to gravity $g_{2}$ and microinertia density $j_{2}$. Meanwhile, $T_{1, w}, T_{2, w}$ and $T_{\infty}$ are temperature of surface and free stream temperature respectively. A sketch of the physical problem is shown in Fig 1. Let $\left(x, y_{1}\right)$ denote the Cartesian coordinates for the upper fluid with $x=0$ as the symmetry plane and $x$ - axis is taken along the interface between the two fluids. It is assumed that the surface is stretched or shrunk with the velocity $u_{w}(x)=a x$, where $a>0$ for a stretching sheet and $a<0$ for a shrinking sheet, respectively. It is also assumed that the constant mass velocity is $v_{0}$, where $v_{0}<0$ for suction and $v_{0}>0$ for injection or withdraw of the fluid, respectively. The coordinate system for the lower fluid is $\left(x, y_{2}\right)$ as shown in Fig. 1 . Note that the $z$-axis is normal to the $\left(x, y_{1}\right)$ plane.

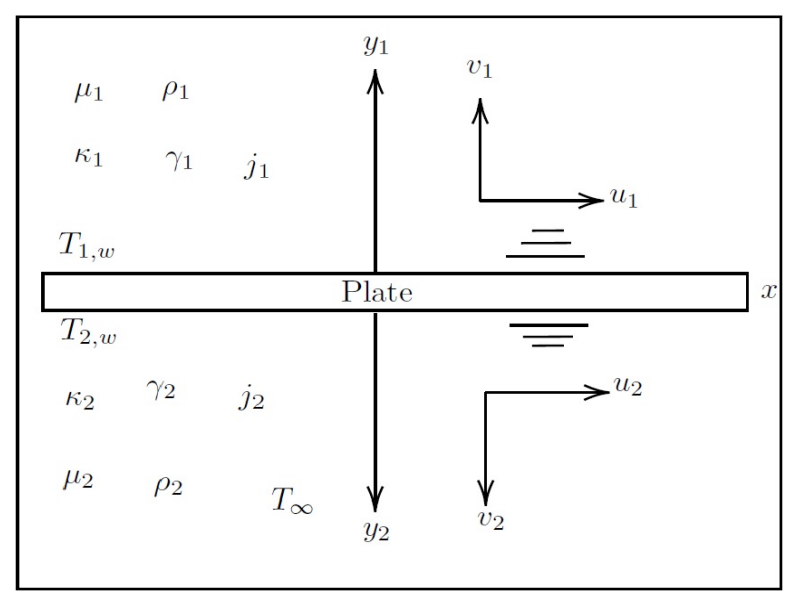

Fig. 1 Physical coordinate.

Under the boundary layer approximation, the governing equations are

$$
\begin{gathered}
\frac{\partial u_{i}}{\partial x}+\frac{\partial v_{i}}{\partial y_{i}}=0 \\
u_{i} \frac{\partial u_{i}}{\partial y_{i}}+v_{i} \frac{\partial u_{i}}{\partial y_{i}}=U_{i} \frac{d U_{i}}{d x}+\frac{\mu_{i}+\kappa_{i}}{\rho_{i}} \frac{\partial^{2} u_{i}}{\partial y_{i}^{2}}+\frac{\kappa_{i}}{\rho_{i}} \frac{\partial N_{i}}{\partial y_{i}} \pm g \beta\left(T_{i}-T_{i, w}\right) \\
\rho_{i} j_{i}\left(u_{i} \frac{\partial N_{i}}{\partial x}+v_{i} \frac{\partial N_{i}}{\partial y_{i}}\right)=\gamma_{i} \frac{\partial^{2} N_{i}}{\partial y_{i}^{2}}-\kappa_{i}\left(2 N_{i}+\frac{\partial u_{i}}{\partial y_{i}}\right) \\
u_{i} \frac{\partial T}{\partial x}+v_{i} \frac{\partial T_{i}}{\partial y_{i}}=\frac{k_{0}}{\rho c_{p}} \frac{\partial^{2} T_{i}}{\partial y_{i}^{2}}
\end{gathered}
$$

subject to boundary conditions

$$
v_{i}=v_{0}, u_{i}(x)=c x, N_{i}=-n \frac{\partial u_{i}}{\partial y_{i}}, T_{i}=T_{i, w} \text { at } y_{i}=0,
$$

$$
u_{1} \rightarrow a x, u_{2}(x) \rightarrow 0, N_{i} \rightarrow 0, T_{i} \rightarrow T_{\infty} \text { at } y_{i} \rightarrow \infty
$$

where $i=1$ is for upper fluid and $i=2$ is for lower fluid. From Jena $\&$ Mathur (1981), the strong concentration case $(n=0)$ represents the concentrated particle flows in which the microelements close to the wall surface are unable to rotate. According to Ahmadi (1976), the weak concentration case $(n=1 / 2)$ indicates the vanishing of the anti-symmetrical part of the stress tensors. The case $n=1$, as suggested by Peddieson (1972), is used for the modelling of turbulent boundary layer flow. In this paper, we consider the cases of $n=0$ (strong concentration) only. Also, we assume that spin-gradient viscosity $\gamma_{i}$ is defined as (Nath, 1975; Rees \& Pop, 1998; Nazar et al., 2004 \& Aurangzaib et al., 2016)

$$
\gamma_{i}=\left(\mu_{i}+\kappa_{i} / 2\right) j_{i}=\mu_{i}(1+K / 2) j_{i} .
$$

Following Attia (2008) for upper fluid, the similarity variables are

$$
\begin{gathered}
u_{1}=a x f^{\prime}(\eta), v_{1}=-\sqrt{a v_{1}} f(\eta), N_{1}=a x \sqrt{a / v_{1}} g(\eta), \\
\eta=y_{1} / \sqrt{v_{1} / a}, \theta_{1}=\frac{T_{1}-T_{\infty}}{T_{1, w}-T_{\infty}}
\end{gathered}
$$

and for lower fluid

$$
\begin{gathered}
u_{2}=a x F^{\prime}(\xi), v_{2}=-\sqrt{a v_{2}} F(\xi), N_{2}=a x \sqrt{a / v_{2}} G(\xi) \\
\xi=y_{2} / \sqrt{v_{2} / a}, \theta_{2}=\frac{T_{2}-T_{\infty}}{T_{2, w}-T_{\infty}},
\end{gathered}
$$

where the prime denotes differentiation with respect to $\eta$ and $\xi$ respectively. Clearly, with $u_{1}$ and $v_{1}$ given in (8), the equation of continuity (1) is satisfied. Similarly, for the lower fluid, with $u_{2}$ and $v_{2}$ given as in (9), it is readily seen that the continuity equation (1) is identically satisfied. Using equations (8) - (9), equations (2) - (6) are transformed into ordinary differential equations for the upper fluid flow

$$
\begin{gathered}
\left(1+K_{1}\right) f^{\prime \prime \prime}+f f^{\prime \prime}-f^{\prime 2}+K_{1} g^{\prime}+1+\theta_{1} \mathrm{Ri}=0, \\
\left(1+K_{1} / 2\right) g^{\prime \prime}+f g^{\prime}-f^{\prime} g-K_{1}\left(2 g+f^{\prime \prime}\right)=0, \\
\theta_{1}{ }^{\prime}+\operatorname{Pr} f \theta_{1}^{\prime}=0,
\end{gathered}
$$

with the boundary conditions

$$
\begin{aligned}
& f(\eta)=\alpha, f^{\prime}(\eta)=\lambda, g(\eta)=-n f^{\prime \prime}(\eta), \theta_{1}(\eta)=1 \text { as } \eta \rightarrow 0, \\
& f^{\prime}(\eta) \rightarrow 1, g(\eta) \rightarrow 0, \theta_{1}(\eta) \rightarrow 0 \text { as } \eta \rightarrow \infty .
\end{aligned}
$$

Next, for the lower fluid, we obtain

$$
\begin{gathered}
\left(1+K_{2}\right) F^{\prime \prime}+F F^{\prime \prime}-F^{\prime 2}+K_{2} G^{\prime}+\theta_{2} \mathrm{Ri}_{2}=0 \\
\left(1+K_{2} / 2\right) G^{\prime \prime}+F G^{\prime}-F^{\prime} G-K_{2}\left(2 G+F^{\prime \prime}\right)=0, \\
\theta_{2}{ }^{\prime \prime}+\operatorname{Pr} f \theta_{2}{ }^{\prime}=0
\end{gathered}
$$

with the boundary conditions 


$$
\begin{aligned}
& F(\xi)=\beta, F^{\prime}(\xi)=\lambda, G(\xi)=-n F^{\prime \prime}(\xi), \theta_{2}(\xi)=1 \text { as } \xi \rightarrow 0, \\
& F^{\prime}(\xi) \rightarrow 0, G(\xi) \rightarrow 0, \theta_{2}(\xi) \rightarrow 0 \text { as } \xi \rightarrow \infty,
\end{aligned}
$$

where Ri is Richardson number, Pr is Prandtl number, $K_{1}$ and $K_{2}$ are micropolar parameters. These quantities can be expressed as

$$
\operatorname{Pr}=\frac{c_{p} \mu_{i}}{\kappa_{i}} \text { and } \mathrm{K}_{i}=\frac{\kappa_{i}}{\mu_{i}}, i=1,2
$$

In addition, we use $\mathrm{Ri}=1$ to indicate mixed convection case.

\section{RESULTS AND DISCUSSION}

Equations (10) to (12) with boundary conditions (13) for upper fluid and equations (14) to (16) with boundary conditions (17) for lower fluid are solved using shooting technique with Runge-Kutta-Gill method. The numerical computation is implemented using Python 3 language in Jupyter Notebook. The graphical representation of velocity, microrotation and temperature are plotted for strong concentration ( $n=0)$ and assisting flow only. According to Yacob \& Ishak (2012), solution for Newtonian fluid $(K=0)$ exists when permeable parameter $\alpha, \beta$ is greater or equal to 2 and as for micropolar fluid $(K>0)$ stronger suction is needed. Therefore, we choose the permeable parameter $\alpha=\beta=3$. In order to verify the present work, we compared the values of skin friction $f^{\prime \prime}(0)$ when stretching parameter $\lambda$ is varies with Rosali et al. (2012) in Table 1 and obtained a good agreement.

Table 1 Comparison of skin friction with a various stretching parameter when $K=0, \operatorname{Pr}=1, \alpha=\beta=0$ and $\mathrm{Ri}=0$

\begin{tabular}{ccc}
\hline$\lambda$ & Rosali et al. (2012) & Present \\
\hline 0 & 1.232588 & 1.232588 \\
0.1 & 1.146561 & 1.146561 \\
0.2 & 1.051130 & 1.051130 \\
0.3 & 0.946816 & 0.946816 \\
0.4 & 0.834072 & 0.834074 \\
0.5 & 0.713295 & 0.713295 \\
1.0 & 0 & 0 \\
2.0 & -1.887307 & -1.887307 \\
3.0 & -4.276541 & -4.276541 \\
4.0 & -7.086378 & -7.086377 \\
5.0 & -10.264749 & -10.264747 \\
\hline
\end{tabular}

Figs. 2 - 4 represent velocity, microrotation and temperature when micropolar parameter varies $\operatorname{Pr}$ number $\operatorname{Pr}=7$, stretching parameter $\lambda=0.5$ and permeable parameter of upper and lower fluid $\alpha=\beta=3$. Fig. 2 shows that the velocity of upper fluid is decreasing while the velocity of lower fluid is increasing as the micropolar parameter increases. Fig. 3 illustrates a similar trend for microrotation. Also, the temperature of the upper fluid is slightly increasing with the addition of micropolar parameter. However, the temperature of lower fluid is decreasing.

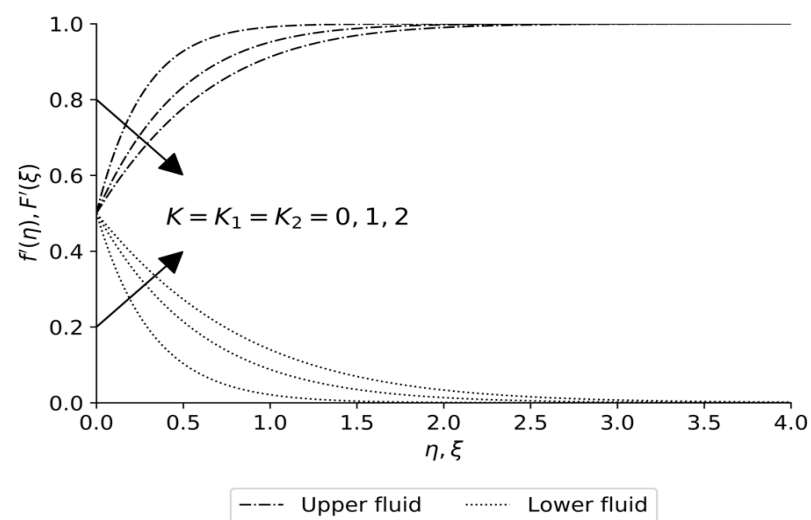

Fig. 2 Velocity profile of upper and lower fluid with a variation of micropolar parameter $K$ when $\operatorname{Pr}=7, \lambda=0.5$ and $\alpha=\beta=3$.

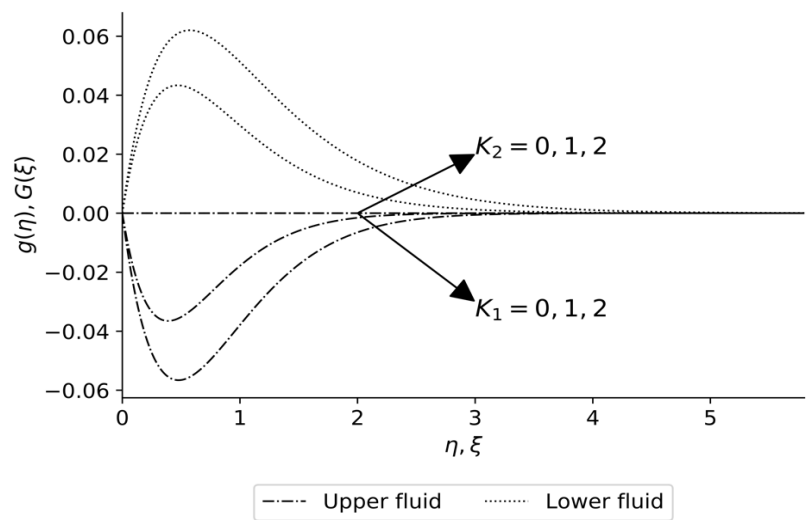

Fig. 3 Microrotation profile of upper and lower fluid with a variation of micropolar parameter $K$ when $\operatorname{Pr}=7, \lambda=0.5$ and $\alpha=\beta=3$.

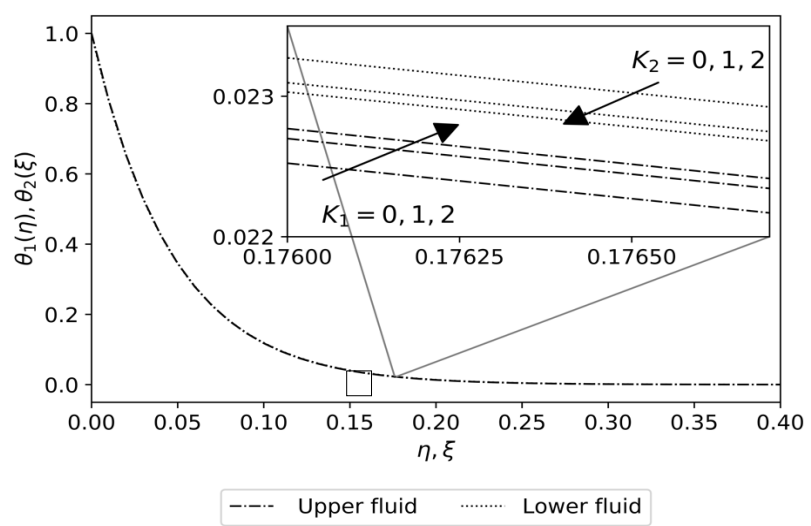

Fig. 4 Temperature profile of upper and lower fluid with a various micropolar parameter $K$ when $\operatorname{Pr}=7, \lambda=0.5$ and $\alpha=\beta=3$.

Figs. $5-7$ are presented to observe the effect of Pr number on velocity, microrotation and temperature when micropolar fluid $K=K_{1}=K_{2}=1$ and stretching parameter $\lambda=0.5$. In real life, $\operatorname{Pr}=0.71$ represents air, $\operatorname{Pr}=1$ indicates an electrolyte solution while $\operatorname{Pr}=7$ is for water (Salleh et al., 2009). It is shown from Fig. 5 that the velocity of upper fluid is decreasing as Pr number increase. On the other hand, the velocity of lower fluid is increasing and has greater boundary layer thickness. Microrotation is following the same trend as seen in Fig. 6. Meanwhile, the temperatures of both upper and lower fluid are is decreasing with the increment of Pr number. Physically, greater Pr number means the fluid has high viscosity which produces less heat while low Pr number indicates that the fluid is highly conductive. 


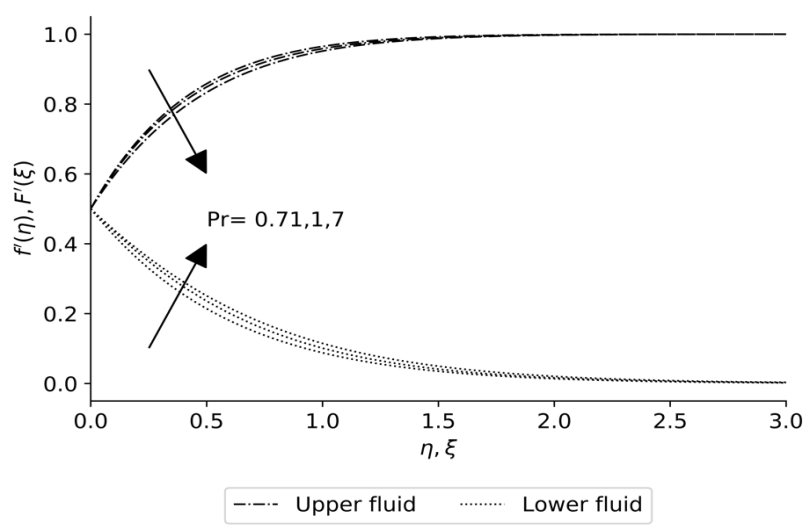

Fig. 5 Velocity profile of upper and lower fluid with a variation of Pr number when $K=K_{1}=K_{2}=1, \lambda=0.5$ and $\alpha=\beta=3$.

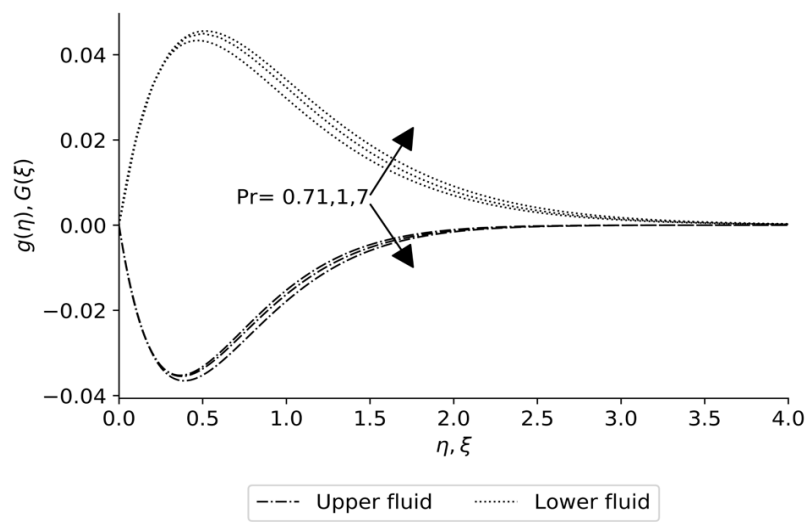

Fig. 6 Microrotation profile of upper and lower fluid with a variation of Pr number when $K=K_{1}=K_{2}=1, \lambda=0.5$ and $\alpha=\beta=3$.

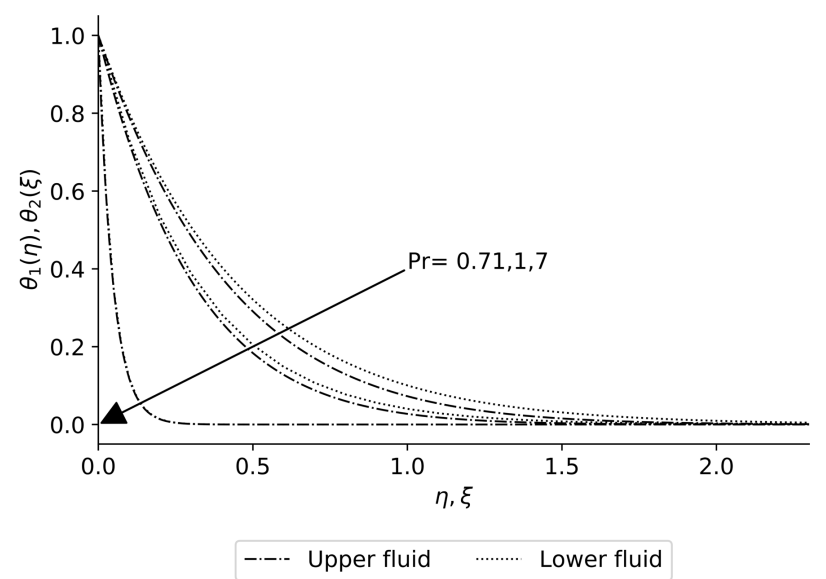

Fig. 7 Temperature profile of upper and lower fluid with a variation of Pr number when $K=K_{1}=K_{2}=1, \lambda=0.5$ and $\alpha=\beta=3$.

Figs. $8-13$ display the effect of stretching parameter on velocity, microrotation and temperature when micropolar $K=K_{1}=K_{2}=1$, $\operatorname{Pr}$ number $\operatorname{Pr}=7$, permeable parameter of upper and lower fluid $\alpha=\beta=3$. From Fig. 8 and Fig. 9, it can be observed that the velocity of upper and lower fluid is increasing as stretching parameter increases. It is seen that at stretching parameter $\lambda=1$, the velocity of upper fluid is constant at 1 as the velocity of stretching surface and velocity of fluid is equal while the velocity of the lower fluid is constantly decreasing toward the boundary. Moreover, the magnitude of microrotation of upper and lower fluid is also increasing with the addition of the stretching parameter as can be seen in Fig. 10 and Fig. 11. The temperature decreases as the stretching parameter increase for both upper and lower fluid. However, it is clear that upper fluid has smaller thermal boundary layer thickness compared to lower fluid.

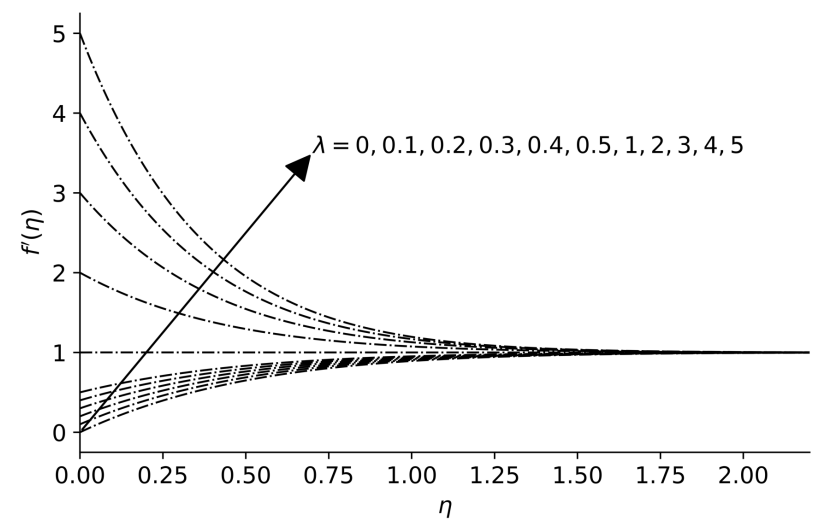

Fig. 8 Velocity profile for upper fluid when stretching parameter $\lambda$ is various with $K=K_{2}=1, \operatorname{Pr}=7$ and $\alpha=\beta=3$.

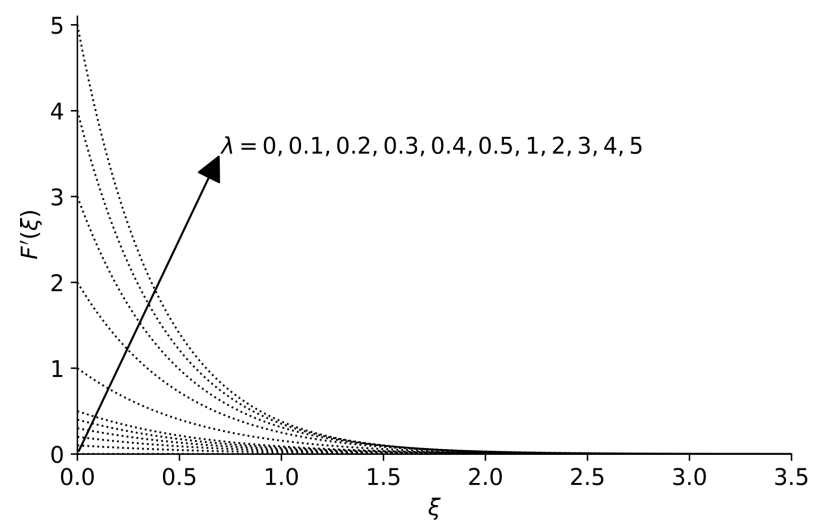

Fig. 9 Velocity profile of lower fluid when stretching parameter $\lambda$ when is various with $K=K_{2}=1, \operatorname{Pr}=7$ and $\alpha=\beta=3$.

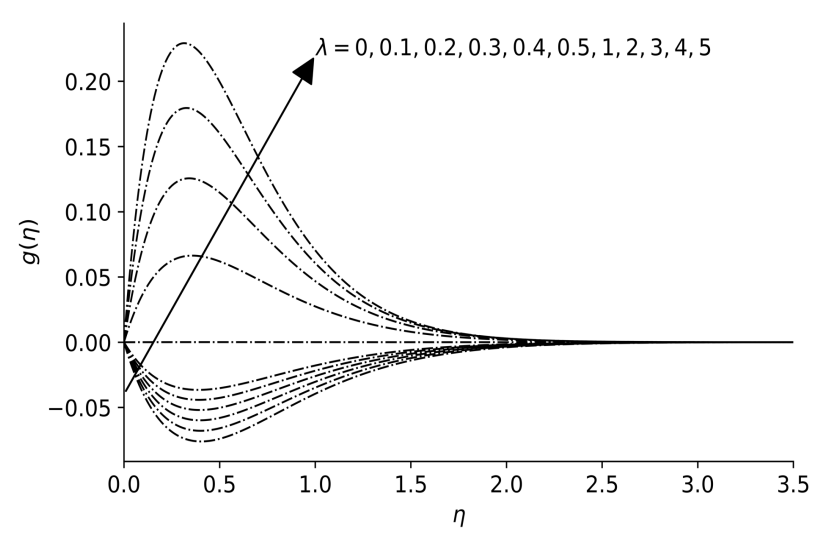

Fig. 10 Microrotation profile for upper fluid when stretching parameter $\lambda$ is various with $K=K_{2}=1, \operatorname{Pr}=7$ and $\alpha=\beta=3$. 


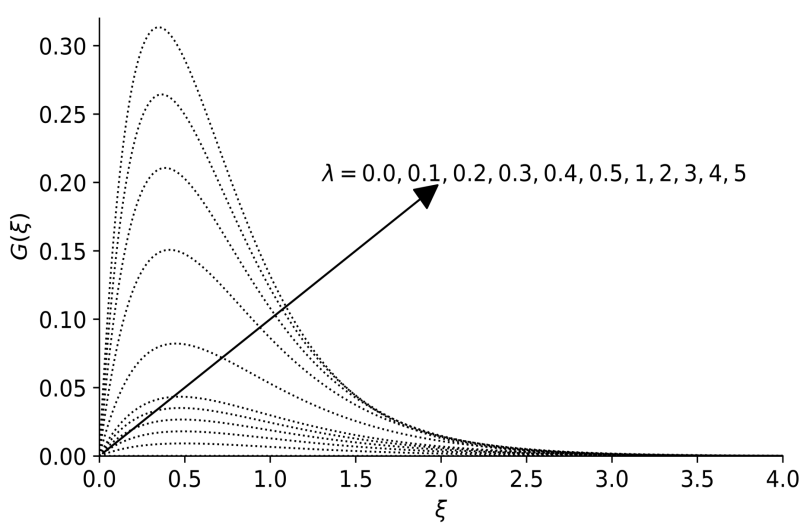

Fig. 11 Microrotation profile for lower fluid when stretching parameter $\lambda$ is various with $K=K_{2}=1, \operatorname{Pr}=7$ and $\alpha=\beta=3$

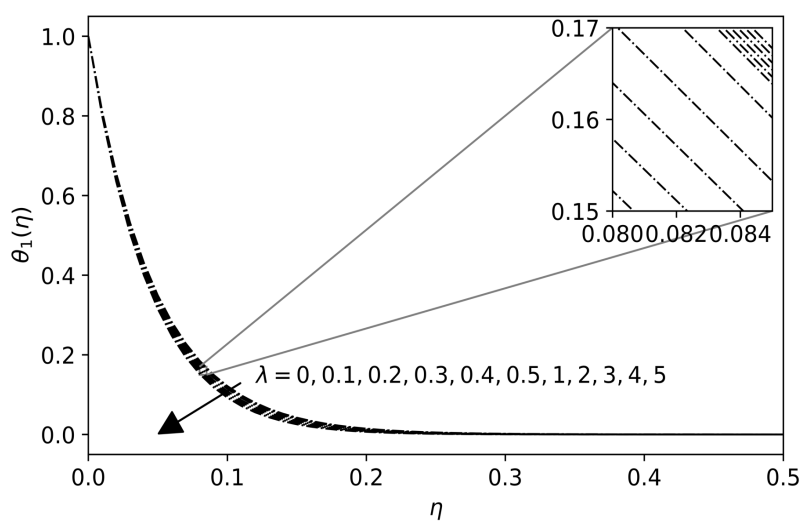

Fig. 12 Temperature profile for upper fluid when stretching parameter $\lambda$ is various with $K=K_{2}=1, \operatorname{Pr}=7$ and $\alpha=\beta=3$.

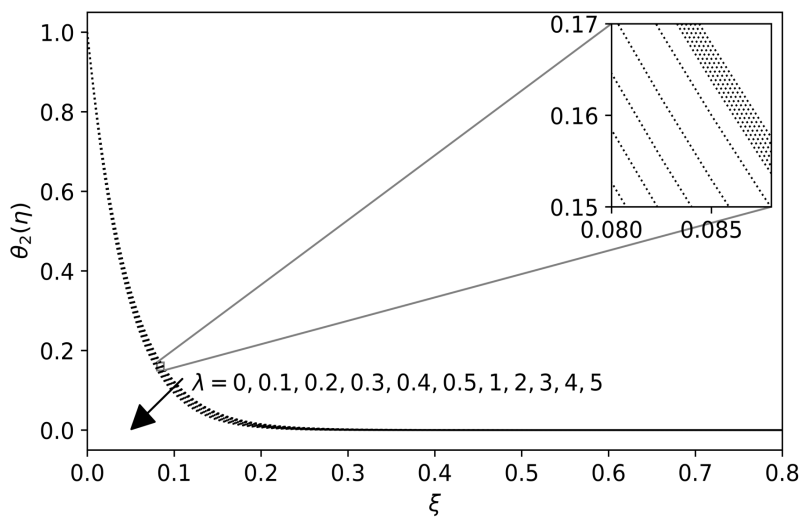

Fig. 13 Temperature profile for lower fluid when stretching parameter $\lambda$ is varied with $K=K_{2}=1, \operatorname{Pr}=7$ and $\alpha=\beta=3$,

Velocity, microrotation and temperature are sketched in Figs 14 16 with permeable parameter of lower fluid greater than upper fluid $\beta>\alpha$, when micropolar parameter $K=K_{1}=K_{2}=1$, Pr number $\operatorname{Pr}=7$ and stretching parameter $\lambda=0.5$. From Fig. 14 , the velocity of upper fluid is slightly increased while the velocity of lower fluid is decreasing with greater boundary layer thickness compared to upper fluid. Next, Fig. 15 exhibits that the microrotation of upper fluid is increasing until it achieves the minimum magnitude then decreasing along with the increment of permeable parameter. Meanwhile, the microrotation of lower fluid is only decreasing after it reaches the peak. Fig 16 presents that the temperature of upper fluid is greater than the temperature of lower fluid. We also noticed that the higher value of permeable parameter, the smaller magnitude of temperature.

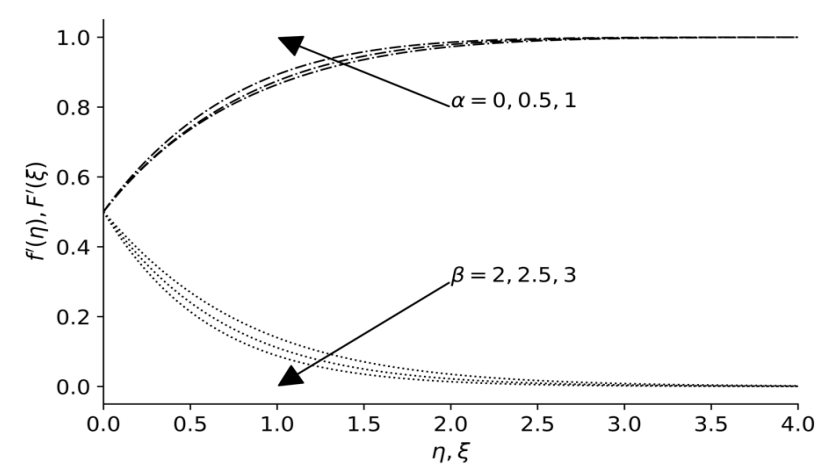

-.-.- Upper fluid .......... Lower fluid

Fig. 14 Velocity profile of upper and lower fluid when permeable parameter is various where $\beta>\alpha$ with $K=K_{1}=K_{2}=1, \operatorname{Pr}=7$ and $\lambda=0.5$.

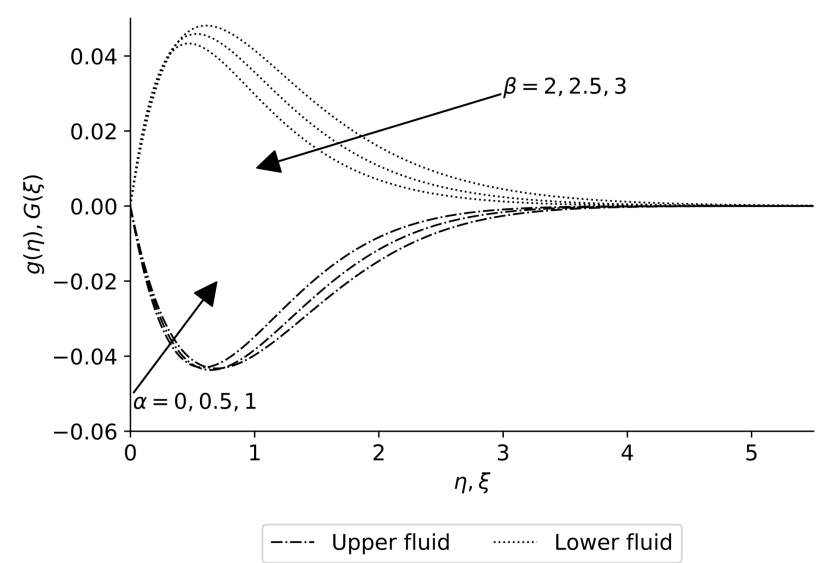

Fig. 15 Microrotation profile of upper and lower fluid when permeable parameter is various where $\beta>\alpha$ with $K=K_{1}=K_{2}=1, \operatorname{Pr}=7$ and $\lambda=0.5$.

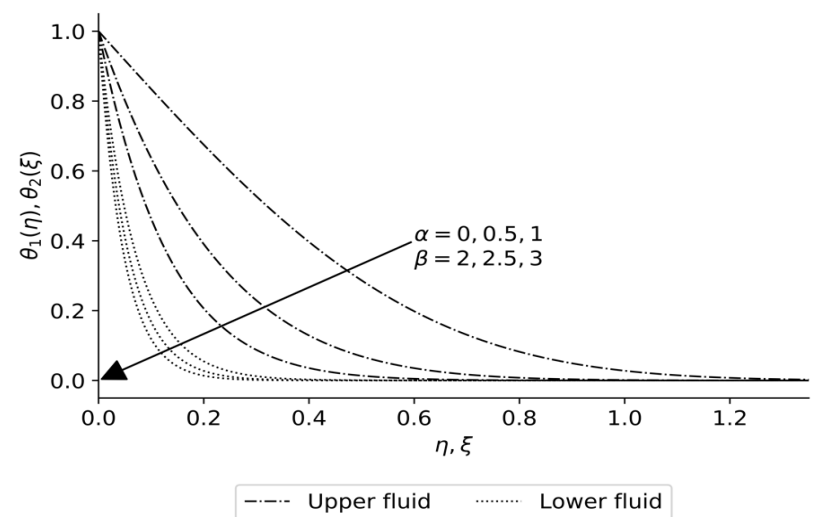

Fig. 16 Temperature profile of upper and lower fluid when permeable parameter is varied where $\beta>\alpha$ with $K=K_{1}=K_{2}=1, \operatorname{Pr}=7$ and $\lambda=0.5$. 


\section{CONCLUSION}

In conclusion, the problem of mixed convection of micropolar fluid flow over a permeable stretching surface on another quiescent fluid is solved. The result shows the velocity of upper fluid is decreasing as micropolar parameter $K$ increases. Meanwhile, the velocity of lower fluid is decreasing with increment of micropolar parameter $K$. We also observed that the temperatures of upper and lower fluid are decreasing as Pr number increases. In addition, we found that the temperature is declining with the addition of permeable parameter $\alpha, \beta$ for both upper and lower fluid.

\section{ACKNOWLEDGEMENT}

The authors would like to acknowledge the Research Management Centre-IIUM (C-RIG18-004-0004) and Research Management Centre-UTM (vote numbers 07G70, 07G72, 07G76 and 07G77) for the financial support provided throughout this research.

\section{REFERENCES}

Ahmadi, G. (1976). Self-similar solution of incompressible micropolar boundary layer flow over a semi-infinite plate. International Journal of Engineering Science, 14(7), 639-646.

Attia, H. A. (2008). Stagnation point flow and heat transfer of a micropolar fluid with uniform suction or blowing. Journal of the Brazilian Society of Mechanical Sciences and Engineering, 30(1), 57-65.

Aurangzaib, Sharif Uddin, M., Bhattacharyya, K., \& Shafie, S. (2016). Micropolar fluid flow and heat transfer over an exponentially permeable shrinking sheet. Propulsion and Power Research, 5(4), 310-317.

Eringen, A. C. (1965). Theory of micropolar fluid. In School of Aeronautics, Astronautice \& Engineering Sciences.

Haghighi, A. R., \& Asl, M. S. (2015). Mathematical modeling of micropolar fluid flow through an overlapping arterial stenosis. International Journal of Biomathematics, 08(04), 1550056.

Ishak, A., Nazar, R., \& Pop, I. (2008). Mixed convection stagnation point flow of a micropolar fluid towards a stretching sheet. Meccanica, 43(4), 411418.

Ishak, Anuar, Nazar, R., Arifin, N. M., \& Pop, I. (2007). Mixed convection of the stagnation-point flow towards a stretching vertical permeable sheet. Malaysian Journal of Mathematical Sciences, 1(2), 217-226.

Jena, S. K., \& Mathur, M. N. (1981). Similarity solutions for laminar free convection flow of a thermomicropolar fluid past a non-isothermal vertical flat plate. International Journal of Engineering Science, 19(11), 14311439.
Kiran, R. G., Radhakrishnamacharya, G., \& Anwar Bég, O. (2017). Peristalsis flow and hydrodynamic dispersion of a reactive micropolar fluidsimulation of chemical effects in the digestive process. Journal of Mechanics in Medicine and Biology, 17(1), 1-17.

Liu, T. (1992). Nonorthogonal stagnation flow on the surface of a quiescent fluid an exact solution of the Navier-Stokes equation. Quarterly of Applied Mathematics, 50(1), 39-47.

Majid, N. A., Mohammad, N. F., Rahman, A., Kasim, M., Ilias, M. R., \& Shafie, S. (2019). Effect of constant heat flux on forced convective micropolar fluid flow over a surface of another quiescent fluid. Universal Journal of Mechanical Engineering, 7(4), 198-205.

Mohamad Isa, S., \& Mohammad, N. F. (2017). Boundary layer flow of dusty on a stretching sheet of another quiescent fluid. Journal of Physics: Conference Series, 819(1).

Nath, G. (1975). Similar solutions for the incompressible laminar boundary layer with pressure gradient in micropolar fluids. Rheologica Acta, 14(9), $850-857$.

Peddieson, J. (1972). An Application of the micropolar fluid model to the calculation of a turbulent shear flow. International Journal Engineering Science, Volume 10(Issue 1), 23-32.

Reza, M., \& Gupta, A. S. (2012). MHD stagnation-point flow of an electrically conducting fluid on the surface of another quiescent fluid. Acta Mechanica, 223(11), 2303-2310.

Reza, M., Panigrahi, S., \& Mishra, A. K. (2017). Stagnation point flow and heat transfer for a viscoelastic fluid impinging on a quiescent fluid. Sadhana - Academy Proceedings in Engineering Sciences, 42(11), 19791986.

Rohni, A. M., Omar, Z., \& Man, N. H. (2015). Dual solutions of stagnationpoint flow of a fluid on a shrinking surface of another quiescent fluid. 4th Int'l Conference on Advances in Engineering Sciences \& Applied Mathematics (ICAESAM'2015), 2(7), 74-76.

Rosali, H., Ishak, A., \& Pop, I. (2012). Micropolar fluid flow towards a stretching/shrinking sheet in a porous medium with suction. International Communications in Heat and Mass Transfer, 39(6), 826-829.

Salleh, M. Z., Nazar, R., \& Pop, I. (2009). Forced convection boundary layer flow at a forward stagnation point with Newtonian heating. Chemical Engineering Communications, 196(9), 987-996.

Takhar, H. S., Agarwal, R. S., Bhargava, R., \& Jain, S. (1998). Mixed convection flow of a micropolar fluid over a stretching sheet. Heat and Mass Transfer, 34(2-3), 213-219.

Wang, C. Y. (1988). Stagnation Flow on the Surface of a Quiescent Fluid - an Exact Solution of the Navier-Stokes Equation. Acta Technica, 33(2), 715730.

Yacob, N. A., \& Ishak, A. (2012). Micropolar fluid flow over a shrinking sheet. Meccanica, 47(2), 293-299. 